\title{
The Kinetics of an Antibiotic Stewardship Intervention: A Quasi-Experimental Study
}

Tali Shafat $\cdot$ Orly Shimoni $\cdot$ Anna Nikonov $\cdot$ Lior Nesher

Received: December 7, 2020 / Accepted: January 13, 2021 / Published online: January 30, 2021

(c) The Author(s) 2021

\section{ABSTRACT}

Introduction: Little is known about the kinetics and different phases of a successful antibiotic stewardship program (ASP) intervention.

Methods: We analyzed the trends of quarterly antibiotic use measured in defined daily dose (DDD)/100 days hospitalization using the Joinpoint Regression Program and interrupted time series analysis to objectively identify shifts in the trends of antibiotic use. We correlated these changes in trends with the introduction of a hospital-wide ASP intervention.

Results: The ASP intervention reduced the overall antibiotic use by $33 \%$, from a prior steady state of $76.5 \mathrm{DDD} / 100$ days hospitalization to a post-intervention steady state of

T. Shafat $\cdot$ L. Nesher $(\varangle)$

Infectious Disease Institute, Soroka University

Medical Center, Beer Sheba, Israel

e-mail: nesherke@bgu.ac.il

T. Shafat · O. Shimoni $\cdot$ L. Nesher

Faculty of Health Sciences, Ben-Gurion University of the Negev, Beer Sheba, Israel

T. Shafat

Clinical Research Center, Soroka University Medical

Center, Beer Sheba, Israel

O. Shimoni · A. Nikonov

Pharmacy Services, Soroka University Medical

Center, Beer Sheba, Israel
51.2 DDD/100 days hospitalization $(p<0.001)$. We identified four distinct phases in the trends: prior steady state (A), early intervention (B), accelerated phase (C), and post steady state (D). From A to B a change of slope $(-1.46)$ [SE 0.37, $95 \%$ CI $-2.23,-0.69(p=0.002)] ; \mathrm{B}$ to $\mathrm{C}$, a further decrease of slope $(-4.70)$ [SE $0.64,95 \%$ CI $-6.03,-3.37(p=0.001)]$; between periods $\mathrm{C}$ and $\mathrm{D}$, straightening out of the slope $(+6.84)$ [SE $0.55,95 \%$ CI 5.70, $7.98(p<0.001)]$ to a new post-intervention steady state. It took 1.5 years after completion of the intervention to reach the new steady state.

Conclusions: We demonstrate that ASP interventions require time to achieve the maximal effect. Successful interventions require physicians to adapt new prescribing behaviors and gain confidence in the change; this adaptation can be a prolonged process and might even take years after the introduction of the ASP. These factors should be considered when planning and implementing ASP interventions.

Keywords: Antibiotics stewardship; Guidelines; Infectious disease; Sustainability 


\section{Key Summary Points}

Antibiotic stewardship interventions are usually introduced gradually while monitoring their effect.

There is little information available on the kinetics of an antibiotic stewardship intervention, and specifically how long it takes for an intervention to reach its maximal effect.

We identified four distinct phases: prior steady state, early intervention, accelerated phase, and post-intervention steady state.

We demonstrate that the maximal effect may take years to achieve after completion of the intervention and current studies may be underestimating effects because of short-term follow-up.

\section{DIGITAL FEATURES}

This article is published with digital features, including a summary slide, to facilitate understanding of the article. To view digital features for this article go to https://doi.org/10.6084/ m9.figshare.13567796.

\section{INTRODUCTION}

Antibiotics are the cornerstone of fighting infections. Their use, however, often has a negative impact on patients and society $[1,2]$. In particular, about $20-50 \%$ of antibiotics used in acute care hospitals are either unnecessary or inappropriate [3]. Hospitals worldwide have implemented antibiotic stewardship programs (ASP) to reduce antibiotic use in the acute care setting [4]. Given that no one intervention fits all problems, ASP guidelines usually recommend choosing and tailoring ASP interventions according to institution-specific problems, setting specific goals that can be objectively measured [5-7]. Furthermore, guidelines recommend a gradual implementation of ASP interventions while monitoring their success at each stage, prior to implementing a new intervention $[5,6]$. While changes in the use of antibiotics do not occur overnight, interventions, per se, also take time to support a change. To date, little is known about the time frame and kinetics for an intervention to reach its maximal effect. We aimed to investigate the time it takes for an intervention to achieve its maximal effect.

\section{METHODS}

\section{Setting}

Soroka University Medical Center is a large referral hospital in southern Israel. A pre-authorization antibiotic restriction policy has been in place for the past 20 years. This policy required paper or phone pre-authorization before use of any parenteral antibiotics, except for penicillin, 1st, 2nd, and 3rd generation cephalosporins, and metronidazole.

\section{Intervention}

In November 2014, an ASP intervention was designed to reduce the overall use of antibiotics, specifically targeting quinolones and beta-lactam/beta-lactamase inhibitors (BLBLI). The intervention included (1) Changing the preauthorization method to a computerized system that required renewal every 3 days. (2) Monitoring of all authorizations by a pharmacist for drug interactions and adjustments for kidney function. (3) Notifying (via daily alerts) infectious disease physicians and department heads about patients treated with antibiotics for more than five consecutive days. No changes were made to the local treatment guidelines, restriction policy, or use due to economic constraints or availability of antibiotics. Educational lectures on ASP were provided with the rollout of the computerized system. The ASP intervention began in Q4 2014, was gradually introduced to the entire hospital, and completed by the end of 
Q4 2015 (15 months later), after which no new intervention was introduced. There was no change in the number of beds (1095), the number of admissions (average of 68,000 a year), or type of patients treated by the hospital during the study period.

\section{Study}

This quasi-experimental study investigated the changes observed during different phases of antibiotic use after introduction of ASP, using STROBE-AMS methodology [8]. The study was approved and the need for informed consent was waived by the Soroka Helsinki ethics research committee (SOR 20-0293).

\section{Data Source}

We collected aggregate data from the organization's management software (Business Intelligence software). The data included information on all adult admissions including the length of admission and antibiotic use in adults. Data time points were defined daily dose (DDD) per 100 days of admission for each 3 months (quarterly) [9]. Pediatric and neonate populations were excluded from this study as the DDD is not an effective method to monitor antibiotic use in this population [10].

\section{Statistical Analysis}

To estimate the changes in quarterly DDD/ 100 days of hospitalization over time, we used the Joinpoint Regression Program and interrupted time series analysis (ITS). Joinpoint Regression Program enabled us to identify the time points where the trend changed. We allowed the software to examine different model options ranging from two to four different time points and our final model used three time points. We confirmed and calculated these changes in trend using ITS, a quasi-experimental approach for evaluating longitudinal effects of interventions. We divided the study followup period into segments of changes in trend, and using an ITS approach, we employed autoregressive integrated moving average
(ARIMA) models to estimate changes in level and trend following the intervention. For simple comparisons of mean quarterly $\mathrm{DDD} /$ 100 days for antibiotic use and comparison of hospital admission numbers, we used Student's $t$ test analysis. For all statistical tests and confidence intervals (CI), as appropriate, $\alpha=0.05$ (two-sided) was used. All $p$ values reported were rounded to three decimal places. Statistical analyses were performed using IBM SPSS Statistics for Windows, version 25.0 (IBM Corp., Armonk, NY, USA) and Joinpoint Regression Program, Version 4.7.0.0-February 2019 (National Cancer Institute, Bethesda, MD, USA).

\section{RESULTS}

Following ASP intervention, average quarterly use of all antibiotics from the pre-ASP steady state to the post-ASP steady state was reduced by $33 \%$, from an average of 76.5 to $51.2 \mathrm{DDD} /$ 100 days hospitalization $(p<0.001)$. Analysis of targeted antibiotics showed a reduction in the use of quinolones by $52 \%$, from 10.2 to 4.9 $(p<0.001)$, and amoxicillin/clavulanate by $64 \%$, from 15.6 to $5.7(p<0.001)$ (Table 1). All other restricted antibiotic usage reduced slightly or did not change significantly. Amongst the non-restricted antibiotics, we observed an increase in the use of 1st and 3rd generation cephalosporins from 0.1 to $3.1(p<0.001)$, and 7.2 to $8.4(p=0.004)$, respectively; a decrease in the use of 2 nd generation cephalosporin from 11.1 to $5.4(p<0.001)$ (Table 1$)$.

The Joinpoint Regression Program identified four distinct periods (Fig. 1): Period A-from Q1/2013 to Q1/2015, representing a steadystate pre-intervention phase; period $\mathrm{B}$-from Q2/2015 to Q3/2016, representing an early intervention phase; period C-from Q4/2016 to Q2/2017, representing an accelerated post-intervention phase; and period D-from Q3/2017 to Q2/2019, representing a steady-state postintervention phase. The number of hospital beds (1095) did not change throughout the study, nor was there a difference in the average quarterly hospitalization days from period A to D $[67,359$ to $69,836,(p=0.93)]$. Using ITS, we compared the different periods by observing 
Table 1 Antibiotic DDD per 100 hospitalization days, per annual quarter, steady-state pre-intervention period (period A) vs. new steady-state post-intervention period (period D)

\begin{tabular}{lccc}
\hline Variable & Pre-intervention (mean \pm SD) & Post-intervention $($ mean \pm SD) & $\boldsymbol{p}$ value \\
\hline Total & $76.5 \pm 3.1$ & $51.2 \pm 1.4$ & $<0.001$ \\
Quinolones & $10.2 \pm 0.8$ & $4.9 \pm 0.3$ & $<0.001$ \\
Amoxicillin/clavulanate & $15.6 \pm 0.9$ & $5.7 \pm 0.4$ & $<0.001$ \\
Piperacillin/tazobactam & $3.0 \pm 0.3$ & $2.0 \pm 0.3$ & $<0.001$ \\
1st generation cephalosporins & $0.1 \pm 0.1$ & $3.1 \pm 0.5$ & $<0.001$ \\
2nd generation cephalosporins & $11.1 \pm 0.9$ & $5.4 \pm 0.8$ & $<0.001$ \\
3rd generation cephalosporins & $7.2 \pm 0.5$ & $8.4 \pm 0.6$ & 0.004 \\
Carbapenems & $1.5 \pm 0.9$ & $1.6 \pm 0.3$ & 0.762 \\
Vancomycin & $1.6 \pm 0.1$ & $1.5 \pm 0.2$ & 0.216 \\
\hline
\end{tabular}

changes in slopes (Fig. 1). Between periods A and $B$, we observed a shift from a mild negative slope of -0.20 to a more profound negative slope of -2.05 , a change of $[-1.46$ ) (SE 0.37, 95\% CI $-2.23,-0.69(p=0.002)$ ]; between periods $\mathrm{B}$ and $\mathrm{C}$, a dramatic deepening of the negative slope to -6.56 , a change of -4.70 [SE $0.64,95 \%$ CI $-6.03,-3.37 \quad(p=0.001)]$; between periods and $\mathrm{D}$, we observed straightening out of the slope to a mild positive slope $(+0.26)$ with a change of slope of +6.84 [SE $0.55,95 \%$ CI 5.70, $7.98(p<0.001)]$, which was maintained as a new steady state.

\section{DISCUSSION}

The results of this study demonstrated that immediately after introducing the ASP intervention, a change in the trend was observed in the hospital's antibiotic use. The negative trend in antibiotic use was maintained at a constant value. Approximately 6 months after the intervention ended and without introduction of any new intervention in that time, we observed an accelerated phase of decrease in antibiotic use until a new steady-state level of antibiotic use was reached. This steady state was achieved approximately 1.5 years after completion of the intervention. The expected time for the success of an ASP intervention has not been addressed in the guidelines. The kinetics of an intervention are a crucial element that should be considered when designing a new ASP intervention. Following the recommendation of most guidelines, which suggest a gradual approach to ASP interventions, giving sufficient time for the initial intervention to succeed would be an ideal approach to further build on top of that success [5]. Furthermore, in many ASP intervention reports, the follow-up period is less than 2 years; according to our results, this can underestimate the intervention effect $[11,12]$. We show that the kinetics of an intervention response is not necessarily linear or immediate. Although an immediate initial response is expected, the accelerated phase can begin months after the introduction or completion of the intervention. Moreover, it could take another year to two before reaching maximal effect and a new steady state is achieved which represents the success as well as sustainability of the intervention.

There may be many reasons for the delay in observing the accelerated phase. This study was not designed to evaluate or understand what modified the physicians' behavior. We hypothesize that the slow pace of change in antibiotic prescribing behavior is probably related to the physicians' confidence in a new practice. The medical community is notoriously resistant to change; previous evidence suggests that successful interventions leading to a change of 


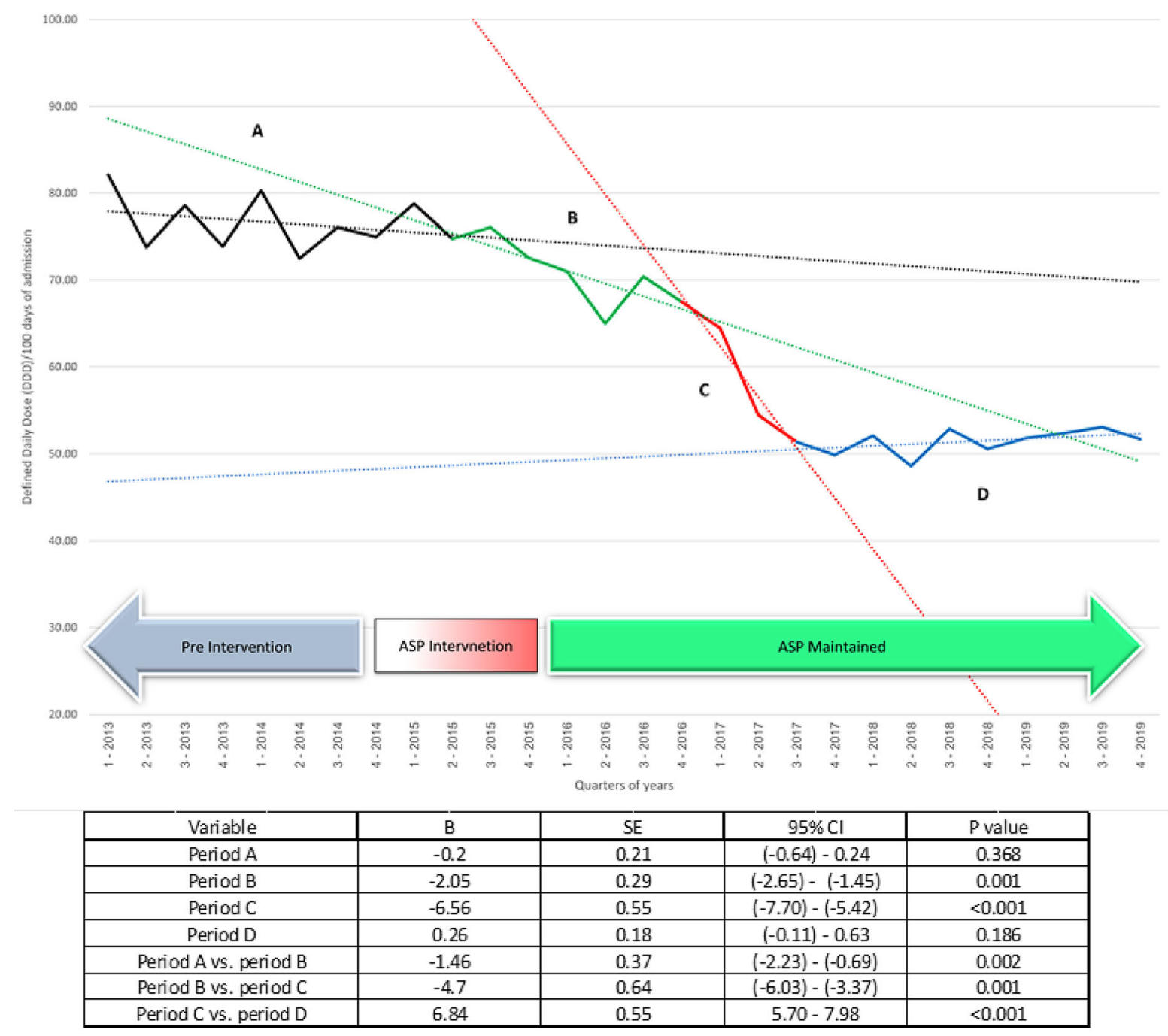

Fig. 1 Quarterly antibiotic use DDD/100 days of admission for all adult patients showing four distinct time periods: a steady-state pre-intervention phase, $\mathbf{b}$ early intervention phase, $\mathbf{c}$ accelerated post-intervention phase, d new steady-state post-intervention phase

which were targeted. Noticeably we observed an increase in the use of cephalosporins, especially the first generation. However, this increase is in our opinion a positive outcome, targeting for example soft tissue infections to be treated with 1st generation cephalosporins and not BLBLI drugs, or treating urinary tract infections with cephalosporins and not with quinolones. As such, this increase in specific antibiotic use represents a welcome effect and not a squeeze the balloon effect as one of the major goals of ASP is to modify treatment to disease-specific reduction of all antibiotics by a significant amount, especially the quinolones and BLBLI 
therapy and not simply overall reduction $[3,17]$.

Limitations to our report include being a single-center study following a multipronged intervention in which we are unable to isolate or identify which element was most significant. Another limitation is the possibility that other factors may have influenced the use of antibiotics that we did not control for, such as changes in pathogen resistance patterns or other possible influences on physicians' behavior. However, this brief report is intended to introduce a novel way to analyze ASP impact on trends of use and shed light on the long-term impact and sustainability of the interventions. Further research is required to assess whether these trend patterns are replicated in other centers and following different types of interventions.

\section{CONCLUSION}

When planning ASP interventions, we recommend considering the kinetics of the intervention, and acknowledging that the time required to reach the maximal effect (a new steady state) may be longer than previously expected.

\section{ACKNOWLEDGEMENTS}

Funding. No funding or sponsorship was received for this study or publication of this article.

Authorship. All named authors meet the International Committee of Medical Journal Editors (ICMJE) criteria for authorship for this article, take responsibility for the integrity of the work as a whole, and have given their approval for this version to be published.

Disclosures. Tali Shafat, Orly Shimoni, Anna Nikonov and Lior Nesher have nothing to disclose.

Compliance with Ethics Guidelines. The study was approved and the need for informed consent was waived by the Soroka Helsinki ethics research committee (SOR 20-0293).

Data Availability. The datasets generated during and/or analyzed during the current study are being used for further research and are available from the corresponding author on reasonable request.

Open Access. This article is licensed under a Creative Commons Attribution-NonCommercial 4.0 International License, which permits any non-commercial use, sharing, adaptation, distribution and reproduction in any medium or format, as long as you give appropriate credit to the original author(s) and the source, provide a link to the Creative Commons licence, and indicate if changes were made. The images or other third party material in this article are included in the article's Creative Commons licence, unless indicated otherwise in a credit line to the material. If material is not included in the article's Creative Commons licence and your intended use is not permitted by statutory regulation or exceeds the permitted use, you will need to obtain permission directly from the copyright holder. To view a copy of this licence, visit http://creativecommons.org/licenses/by$\mathrm{nc} / 4.0 /$.

\section{REFERENCES}

1. Baur D, Gladstone BP, Burkert F, et al. Effect of antibiotic stewardship on the incidence of infection and colonisation with antibiotic-resistant bacteria and Clostridium difficile infection: a systematic review and meta-analysis. Lancet Infect Dis. 2017;17:990-1001. https://doi.org/10.1016/S14733099(17)30325-0.

2. Blaser MJ. Antibiotic use and its consequences for the normal microbiome. Science. 2016;80(352): 544-5. https://doi.org/10.1126/science.aad9358.

3. Davey P, Marwick CA, Scott CL, et al. Interventions to improve antibiotic prescribing practices for hospital inpatients. Cochrane Database Syst Rev. 2017. https://doi.org/10.1002/14651858.CD003543. pub4.

4. Schuts EC, Hulscher MEJL, Mouton JW, et al. Current evidence on hospital antimicrobial 
stewardship objectives: a systematic review and meta-analysis. Lancet Infect Dis. 2016;16:847-56. https://doi.org/10.1016/S1473-3099(16)00065-7.

5. Pollack LA, Srinivasan A. Core elements of hospital antibiotic stewardship programs from the Centers for Disease Control and Prevention. Clin Infect Dis. 2014;59:S97-100. https://doi.org/10.1093/cid/ ciu542.

6. Barlam TF, Cosgrove SE, Abbo LM, et al. Implementing an antibiotic stewardship program: guidelines by the Infectious Diseases Society of America and the Society for Healthcare Epidemiology of America. Clin Infect Dis. 2016;62:e51-77. https://doi.org/10.1093/cid/ciw118.

7. Tamma PD, Avdic E, Keenan JF, et al. What is the more effective antibiotic stewardship intervention: preprescription authorization or postprescription review with feedback? Clin Infect Dis. 2017;64: 537-43. https://doi.org/10.1093/cid/ciw780.

8. Tacconelli E, Cataldo MA, Paul M, et al. STROBEAMS: recommendations to optimise reporting of epidemiological studies on antimicrobial resistance and informing improvement in antimicrobial stewardship. BMJ Open. 2016;6:e010134. https:// doi.org/10.1136/bmjopen-2015-010134.

9. Morris AM. Antimicrobial stewardship programs: appropriate measures and metrics to study their impact. Curr Treat Options Infect Dis. 2014;6: 101-12. https://doi.org/10.1007/s40506-014-00153.

10. Sadasivam B, Malik S, Atal S, Ahmed SN. Development and validation of a mathematical model to quantify antibiotic consumption in paediatric population: a hospital-based pilot study. J Clin Pharm Ther. 2020;45:1349-56. https://doi.org/10. $1111 /$ jcpt.13216.
11. Rahal JJ, Urban C, Horn D, et al. Class restriction of cephalosporin use to control total cephalosporin resistance in nosocomial Klebsiella. J Am Med Assoc. 1998;280:1233-7. https://doi.org/10.1001/jama. 280.14 .1233 .

12. Jenkins TC, Knepper BC, Sabel AL, et al. Decreased antibiotic utilization after implementation of a guideline for inpatient cellulitis and cutaneous abscess. Arch Intern Med. 2011;171:1072-9. https:// doi.org/10.1001/archinternmed.2011.29.

13. Chauhan BF, Jeyaraman M, Mann AS,et al. Behavior change interventions and policies influencing primary healthcare professionals' practice-an overview of reviews. Implement Sci. 2017. https://doi.org/10. 1186/s13012-016-0538-8.

14. Tu K, Davis D. Can we alter physician behavior by educational methods? Lessons learned from studies of the management and follow-up of hypertension. J Contin Educ Health Prof. 2002;22:11-22. https:// doi.org/10.1002/chp.1340220103.

15. Wakefield JG. Commitment to change: exploring its role in changing physician behavior through continuing education. J Contin Educ Health Prof. 2004;24:197-204. https://doi.org/10.1002/chp. 1340240403.

16. Claeys KC, Hopkins TL, Vega AD, Heil EL. Fluoroquinolone restriction as an effective antimicrobial stewardship intervention. Curr Infect Dis Rep. 2018. https://doi.org/10.1007/s11908-018-0615-z.

17. Foolad F, Nagel JL, Eschenauer G, Patel TS, Nguyen CT. Disease-based antimicrobial stewardship: a review of active and passive approaches to patient management. J Antimicrob Chemother. 2017;72: 3232-44. https://doi.org/10.1093/jac/dkx266. 